\title{
Continuous Photo-Oxidation of Fulvenes in a Continuous Flow Photoreactor using Carbon Dioxide as a Solvent
}

Lingqiao Wu, ${ }^{[a]}$ Darren S. Lee, ${ }^{*[a]}$ Hamza Boufroura, ${ }^{[a]}$ Martyn Poliakoff, ${ }^{*[a]}$ and Michael W. George*[a,b]

[a] School of Chemistry, University of Nottingham, University Park, NG7 2RD, UK

[b] Department of Chemical and Environmental Engineering, University of Nottingham Ningbo China, 199 Taikang East Road, Ningbo 315100, China

Email: darren.lee@nottingham.ac.uk; martyn.poliakoff@nottingham.ac.uk; mike.george@nottingham.ac.uk.

\begin{abstract}
A range of 3-substituted oxepinones have been synthesised by the photo-oxidation of fulvenes with photochemically generated singlet oxygen $\left({ }^{1} \mathrm{O}_{2}\right)$ using carbon dioxide $\left(\mathrm{CO}_{2}\right)$ as a solvent and a $\mathrm{CO}_{2}$-soluble porphyrin photosensitizer. The reactive intermediates generated in this reaction decompose to yield a range of useful and underutilized building blocks that are not easily accessible using more traditional reaction setups. Fulvenes containing an exocyclic allylic hydrogen are shown to form three products via potentially competing intramolecular pathways, which can be tuned to some extent by adjusting the conditions in the flow reactor. A range of substrates containing a variety of functional groups was examined; this led to lower yields of the desired lactones being observed in the case of compounds containing polar electron-donating groups. We also demonstrate that one of the lactones can be further hydrogenated over a Pd/C catalyst to the unsaturated 7-membered ring in high yield.
\end{abstract}




\section{Introduction}

The photochemical generation of singlet oxygen $\left({ }^{1} \mathrm{O}_{2}\right)$ from molecular oxygen $\left(\mathrm{O}_{2}\right)$ is a useful method for incorporating oxygen into molecules, ${ }^{[1-4]}$ and the current resurgence of interest towards photochemistry means that reactions involving singlet oxygen are the focus of many research groups ${ }^{[5]}$ and pharmaceutical companies. ${ }^{[6]}$ Singlet oxygen is a short-lived reagent that reacts readily with electron-rich systems forming peroxides in a [2+2] or a [2+4] fashion; furthermore the Schenk-ene reaction is also possible for allylic systems.

Reactions involving photochemically generated ${ }^{1} \mathrm{O}_{2}$ have traditionally been problematic during scale up as larger vessels suffer from poorer light penetration and inefficient irradiation, furthermore there is often the requirement to use pressurised sources of oxygen or air. There are several approaches for addressing the scale-up problems by using ${ }^{1} \mathrm{O}_{2}$ in continuous flow, including falling film reactors, ${ }^{[7]}$ nebulizer flow reactor, ${ }^{[8]}$ gas-liquid membrane reactors, ${ }^{[9]}$ and others. ${ }^{[10-12]}$ One of the main focuses of our research group has been the development of photochemical reactors that use either liquid $\mathrm{CO}_{2}\left(\mathrm{liqCO}_{2}\right)$ or supercritical $\mathrm{CO}_{2}\left(\mathrm{scCO}_{2}\right)$ as the reaction solvent. ${ }^{[13-18]}$ Using $\mathrm{CO}_{2}$ for photo-oxidation reactions has several advantages since $\mathrm{CO}_{2}$ is fully oxidized, is non-flammable and is completely miscible with $\mathrm{O}_{2}$; however the system must be pressurized to maintain the $\mathrm{CO}_{2}$ in the liquid or supercritical phase. In addition, singlet oxygen has also been shown to have a long lifetime in $\mathrm{scCO}_{2}(5.1 \mathrm{~ms}$ at $314 \mathrm{~K}$ and 147 bar) ${ }^{[19]}$ compared to many organic solvents.

Peroxides formed from the reaction of ${ }^{1} \mathrm{O}_{2}$ are often reactive species that can be easily transformed further into more stable compounds of synthetic interest. Recently, we reported the photo-oxidation of cyclopentadiene using liq $\mathrm{CO}_{2}$ and $\mathrm{ScCO}_{2}$ in a pressurized continuous flow system. ${ }^{[20]}$ The endoperoxide formed in this reaction is unstable and can be difficult to handle in large quantities. Therefore, we used liqCO $\mathrm{CO}_{2}$ and $\mathrm{scCO}_{2}$ to produce a modular engineering approach with different quench reagents that could be fed in downstream of the photoreactor, allowing access to a choice of four different compounds.

Continuous flow offers several advantages when generating compounds that are either highly reactive or unstable. ${ }^{[21]}$ The reactors are typically of small volume; therefore only small amounts of the hazardous material are generated at any one time. Reactors and systems can easily be engineered to allow for the addition of quench streams or reagent feeds meaning that unstable intermediates can quickly be reacted to form more stable compounds. Furthermore, parameters such as temperature and pressure can be monitored at multiple points throughout the system and safety cut-offs can be built in so that the system shuts down automatically if a problem arises. 
Fulvenes (1) are closely related in structure to cyclopentadiene and hence the photo-oxidation of such molecules also leads to unstable peroxide intermediates (Scheme 1b). The endoperoxide (I) of fulvene derivatives decompose via a radical based opening of the peroxide to yield a Z-enal containing an epoxide moiety (II) in a way similar to cyclopentadiene (Scheme 1). ${ }^{[22]}$ The additional functionality of fulvenes leads to further decomposition products (III) which ultimately yields a 7-membered lactone containing a Z,Z-diene (2). Previous reports of these reactions and the decomposition products have primarily been performed on small or analytical scales. ${ }^{[23-27]}$ In this paper, we apply our high pressure continuous photoreactor to the photo-oxidation of fulvenes to exploit this relatively under-used transformation enabling the production of potentially scalable quantities of these useful synthetic building blocks.

a)

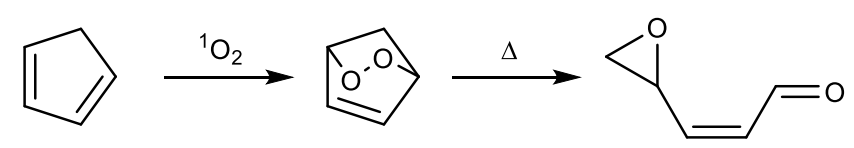

b)

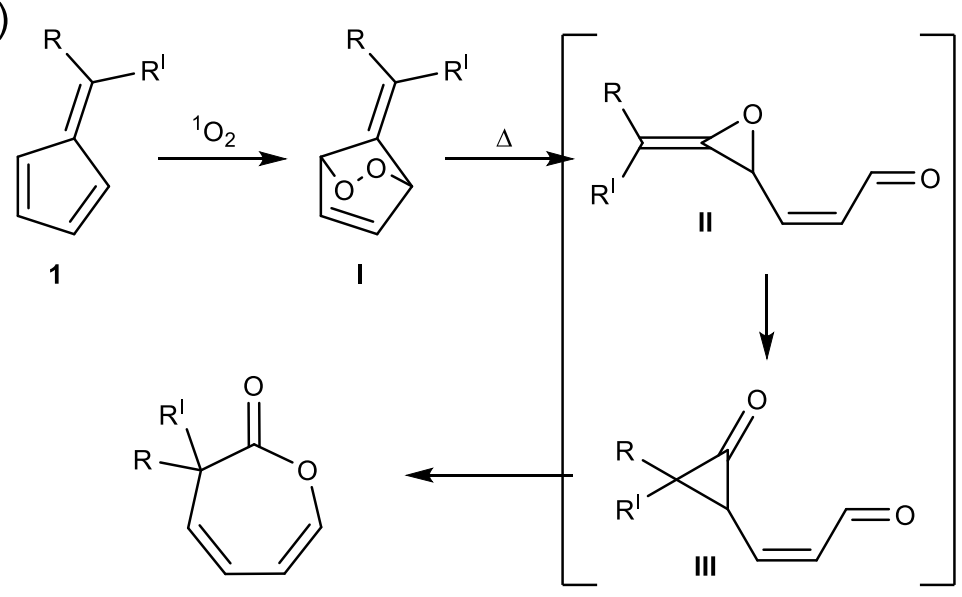

2

Scheme 1. The photo-oxidation and thermal degradation products of a) cyclopentadiene and b) fulvenes.

\section{Results and Discussion}

The high pressure equipment used in this study has been described previously. ${ }^{[20]}$ Such equipment should be used with caution and only with the appropriate safety measures in place. A simplified diagram of the setup is shown in Figure 1. Here, we premix the $\mathrm{O}_{2}$ with $\mathrm{CO}_{2}$ before it is brought into contact with the organic stream to prevent building up a high concentration of oxygen in the presence of the flammable organic stream. 


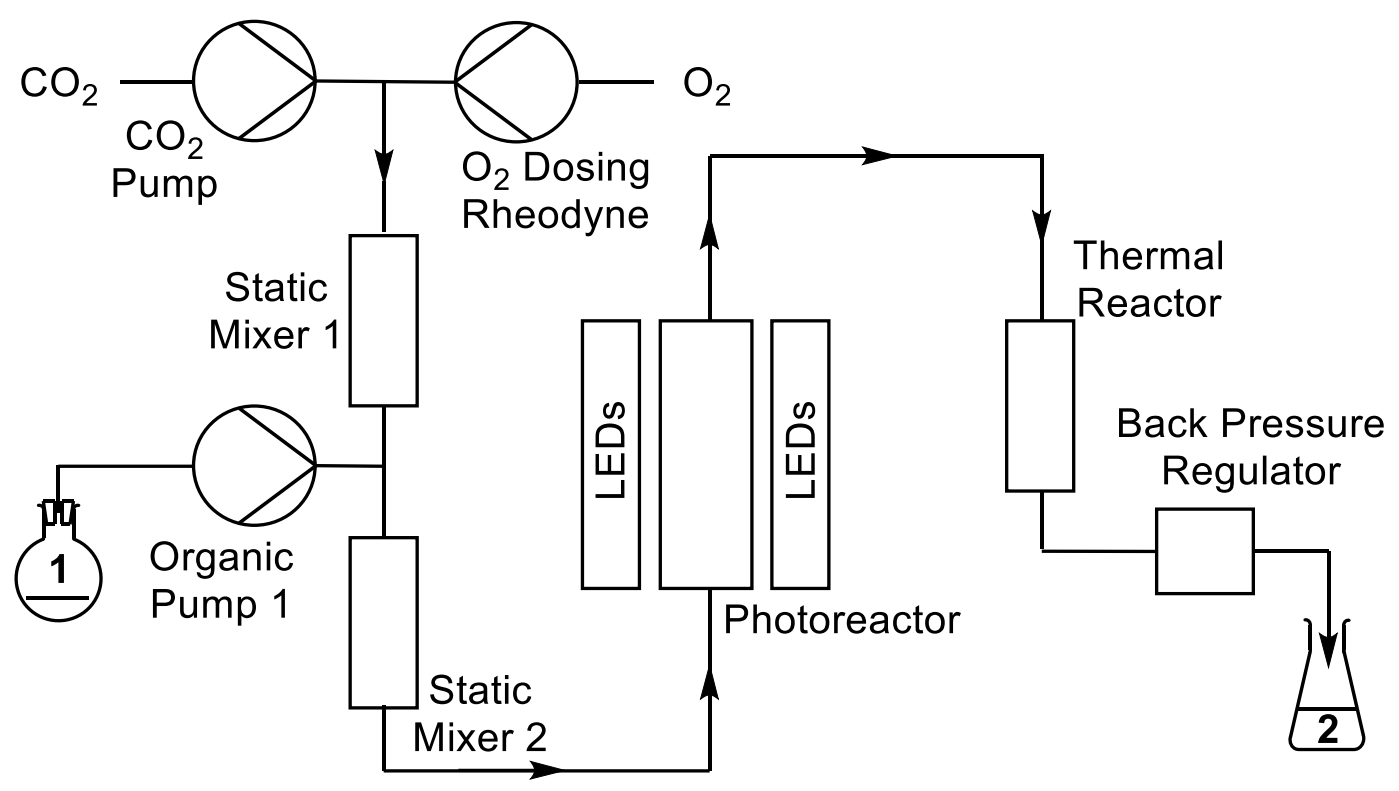

Figure 1. Simplified schematic of the high pressure continuous flow setup. ${ }^{\left[{ }^{[0]}\right.} \mathrm{CO}_{2}$ is delivered from a chilled $\mathrm{CO}_{2}$ pump and $\mathrm{O}_{2}$ is dosed at a known rate via a Rheodyne dosage unit. The gases are then mixed in a stainless steel tubular reactor filled with sand (Static Mixer 1), before the organic reagents are delivered and the components mixed again (Static Mixer 2; consisting of a stainless steel tube filled with sand). Organic Pump 1 is an HPLC pump which delivers a solution of photosensitizer and fulvene. The Photoreactor consists of an upward flow sapphire tube which is filled with glass balls ( $6 \mathrm{~mm}$ diameter) and is irradiated from the outside using white light LEDs. Downstream of the Photoreactor is a Thermal Reactor, consisisting of four stainless steel tubular reactors linked in series and housed in an aluminium heating block. The Back Pressure Regulator is used to maintain the system pressure and to release the reaction mixture continuously. Further details are provided in the ESI.

We began with 6,6-dimethyl fulvene $\mathbf{1 a}$ as the model substrate because this fulvene is a liquid at ambient temperature and can be synthesised quickly on a large scale from acetone and cyclopentadiene. ${ }^{[28,29]}$ Our starting conditions were those which we reported for the photooxidation of cyclopentadiene. ${ }^{[20]}$ The $\mathrm{CO}_{2}$-soluble photosensitizer TPFPP $(5,10,15,20$-tetrakis(pentafluorophenyl)porphyrin) was chosen as the catalyst for the reaction as this has been successfully employed in our system before. ${ }^{[18]}$ As $1 \mathrm{a}$ is quite a viscous oil, a co-solvent was used so that the mixture could be pumped with minimal blockages; initially methanol was chosen. Experiments with 1a in methanol led to a mixture of products with the expected lactone $\mathbf{2} \mathbf{a}$ as the major component (Table 1 ). Adjusting the conditions of the flow reactor had some effect on the observed ratios of $\mathbf{2 a : 3 : 4 a , ~ a s ~ h i g h l i g h t e d ~ i n ~ T a b l e ~ 1 . ~ I n c r e a s i n g ~ t h e ~}$ concentration to $1 \mathrm{M}$ gave a slight drop in conversion (94\%) and decreased the amount of $\mathbf{2 a}$ 
formed (Table 1, entry 2). Decreasing the temperature of the photoreactor and thermal reactor led to a drop in the yield of $\mathbf{2 a}$, with a slight increase in yield of $\mathbf{3}$ and a decrease in the amount of 4 (Table 1, entry 3). Diluting the reaction mixture by increasing the $\mathrm{CO}_{2}$ flow rate (and hence lowering the concentration of 1 in the reactor) increased the yield of $2 a$ to $52 \%$ and $55 \%$ at 0.5 $\mathrm{mL} \mathrm{min}^{-1}$ and $0.75 \mathrm{mLmin}^{-1}$ respectively (Table 1, entries 4-5). Reducing the concentration of 1 in $\mathrm{MeOH}$ also improved the yield and selectivity toward $2 \mathrm{a}$ (Table 1, entry 6). Switching to an aprotic solvent appeared to supress the formation of $\mathbf{3}$ and led to a slight increase in the yield of $2 a$ (Table 1 , entry 7 ). Increasing the reactor temperature to $80{ }^{\circ} \mathrm{C}$ gave a further increase in the yield of $2 \mathrm{a}$ to $66 \%$ (Table 1 , entry 8 ).

Table 1. Conditions affecting the ratio of formation of $\mathbf{2 a}, \mathbf{3}$ and $\mathbf{4}$ from $\mathbf{1 a}$. $^{\mathrm{a}}$<smiles>CC(C)=C1C=CC=C1</smiles>

$1 \mathbf{a}$

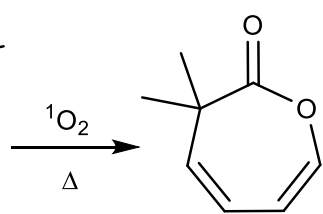

$\mathbf{2 a}$

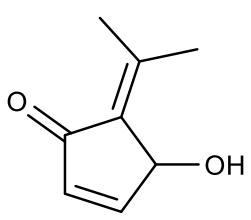

3

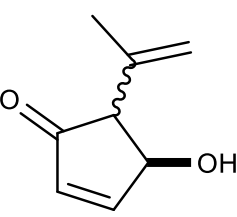

$4 a$

\begin{tabular}{|c|c|c|c|c|c|c|c|}
\hline \multirow[t]{2}{*}{ Entry } & \multirow{2}{*}{$\begin{array}{l}\text { Conc. } \\
\text { (M) }\end{array}$} & \multirow{2}{*}{$\begin{array}{c}\mathrm{CO}_{2} \text { Flow Rate } \\
\left(\mathrm{mL} \mathrm{min}^{-1}\right)\end{array}$} & \multirow{2}{*}{$\begin{array}{l}\text { Temp. } \\
\left({ }^{\circ} \mathrm{C}\right)^{\mathrm{b}}\end{array}$} & \multirow{2}{*}{$\begin{array}{c}\text { Co- } \\
\text { solvent }\end{array}$} & \multicolumn{3}{|c|}{ Yield $(\%)^{c}$} \\
\hline & & & & & $2 a$ & 3 & $4 a$ \\
\hline 1 & 0.1 & 0.25 & $40 / 40$ & $\mathrm{MeOH}$ & 37 & 14 & 49 \\
\hline $2^{d}$ & 1.0 & 0.25 & $40 / 40$ & $\mathrm{MeOH}$ & 28 & 13 & 35 \\
\hline 3 & 0.1 & 0.25 & $20 / 20$ & $\mathrm{MeOH}$ & 34 & 17 & 34 \\
\hline 4 & 0.1 & 0.5 & $40 / 40$ & $\mathrm{MeOH}$ & 52 & 10 & 33 \\
\hline 5 & 0.1 & 0.75 & $40 / 40$ & $\mathrm{MeOH}$ & 55 & 8 & 22 \\
\hline 6 & 0.05 & 0.5 & $40 / 40$ & $\mathrm{MeOH}$ & 76 & 22 & $<1$ \\
\hline 7 & 0.1 & 0.75 & $40 / 40$ & EtOAc & 57 & - & 18 \\
\hline 8 & 0.1 & 0.75 & $40 / 80$ & EtOAc & 66 & - & 14 \\
\hline $9^{e}$ & 0.25 & 0.75 & $40 / 40$ & EtOAc & 44 & 4 & $<1$ \\
\hline
\end{tabular}

a) $0.1 \mathrm{~mol}-\%$ TPFPP as a catalyst, Organic flow rate $=0.1 \mathrm{~mL} \mathrm{~min}^{-1}$, System pressure $=120$ bar. b) Photoreactor/Thermal reactor temperatures. c) Unless stated, conversion >99\%; conversion and yield were measured by ${ }^{1} \mathrm{H}$ NMR using mesitylene as an internal standard. d) Conversion $=94 \%$. e) Starting mixture contained 1 equiv. $\mathrm{NEt}_{3}$, conversion $=81 \%$.

The formation of $\mathbf{4 a}$ as a ca. 1:1 mixture of diastereoisomers was observed in all reactions starting from 1a regardless of the solvent choice, suggesting that its formation is not an intermolecular process. Instead, $\mathbf{4 a}$ is likely to be formed from a competing rearrangement facilitated by intramolecular hydrogen atom transfer (Scheme 2). Decomposition of 
endoperoxide I forms the biradical intermediate IV which can then proceed through one of two competing pathways. Pathway A is dominant, with formation of aldehyde II and III via cleavage of a C-C bond in the cyclopentene ring (Scheme 2, shown with red arrows). Pathway B (Scheme 3, shown with black arrows) proceeds via abstraction of an allylic hydrogen atom and rearrangement to form the bicyclic epoxide intermediate $\mathbf{V}$ which should then yield a single diastereoisomer based on the stereochemistry setup by addition of singlet oxygen. A related 1,7-hydrogen atom transfer (HAT) mechanism and the isolation of compounds similar to $\mathbf{V}$ have recently been reported ${ }^{[23]}$ for oxygenated fulvene derivatives, which lends support for this hypothesis. Intermediate $\mathbf{V}$ was neither isolated nor observed by ${ }^{1} \mathrm{H}$ NMR in any of our experiments. In this case, the formation of $\mathbf{4 a}$ from $\mathbf{V}$ probably proceeds via a hydrogen transfer mechanism that racemises the chiral centre. A possible cause for this is that this hydrogen atom transfer event proceeds primarily via an intermolecular process, perhaps involving a second molecule of $\mathbf{V}$ (or involving methanol when it is used as the solvent) acting as a hydrogen atom donor/acceptor. It is also possible that the conversion of IV to $\mathbf{V}$ is reversible because, when the reaction is diluted with $\mathrm{CO}_{2}$ and an aprotic co-solvent used, 2a is the major product and, in this case, it is likely that the conversion of $\mathbf{V}$ to $\mathbf{4 a}$ is impeded. Further experiments to clarify this mechanism are currently in progress.

The minor product $\mathbf{3}$ is a motif that is often obtained from treatment of a cyclic endoperoxide with a catalytic base. ${ }^{[30,31]}$ In the case of 1 a where no base is added, it may be that methanol is involved in the formation of $\mathbf{3}$ from endoperoxide I or from the bi-radical intermediate IV. The exact mechanism, whether it is an ionic or radical based one, is currently unknown. When methanol was replaced with an aprotic solvent such as ethyl acetate or toluene, the formation of $\mathbf{3}$ was supressed to a level that it was not detectable in the product mixture. Addition of $\mathrm{NEt}_{3}$ $(0.25 \mathrm{M} ; 1$ equiv.) to the reaction mixture, in attempt to tune selectivity towards $\mathbf{3}$, resulted in a loss of efficiency for the photo-oxidation and some polymerisation, however 2 a was still observed as the major product (Table 1 , entry 9). ${ }^{[32]}$ 


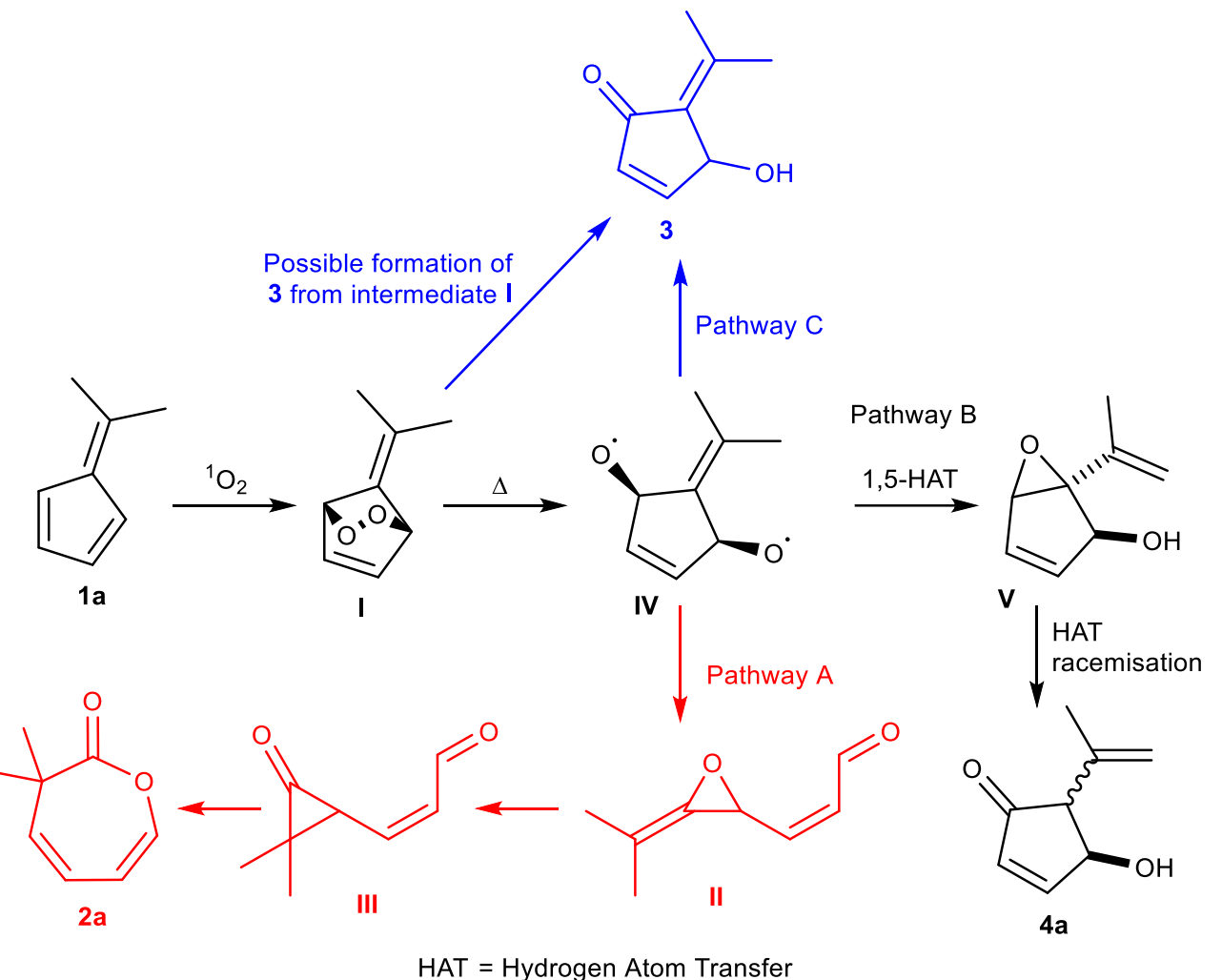

Scheme 2. Suggested competing mechanistic pathways for the formation of $2 a, 3$ and $\mathbf{4}$ from 1a.

Given that there are several competing pathways when reacting $1 \mathrm{a}$ with ${ }^{1} \mathrm{O}_{2}$, moving to a substrate that lacks a labile allylic hydrogen atom should inhibit this competing pathway and give only the lactone product selectively. Therefore, several different fulvenes were synthesised and subjected to the flow reactor to probe their reactivity (Scheme 3). Using a similar substrate to $\mathbf{1 a}$, the cyclohexyl derivative $\mathbf{1 b}$ formed bicyclic lactone $\mathbf{2} \mathbf{b}$ containing a spiro centre as the major product and formed the hydroxyenone $4 \mathrm{~b}$ (up to $48 \%$ by ${ }^{1} \mathrm{H} N M R$ ) as a competing product (in all cases $\mathbf{4 b}$ could not be isolated and appeared to decompose in the presence of $\mathrm{SiO}_{2}$ or $\mathrm{Al}_{2} \mathrm{O}_{3}$ ). A substrate with a more hindered allylic proton (1c) yielded exclusively the lactone (2c) and no competing products were observed, suggesting that suppression of the hydrogen abstraction pathway was occurring. When substrates contained aromatic groups were used, the results were varied. With the fulvene derived from benzaldehyde (1d), the lactone was obtained as the major product in $70 \%$ yield. When an aromatic substrate containing an electron withdrawing group (1e) was reacted, the conversion of the fulvene remained high; however, the overall yield of the lactone suffered (37\% for $\mathbf{2 e})$. 

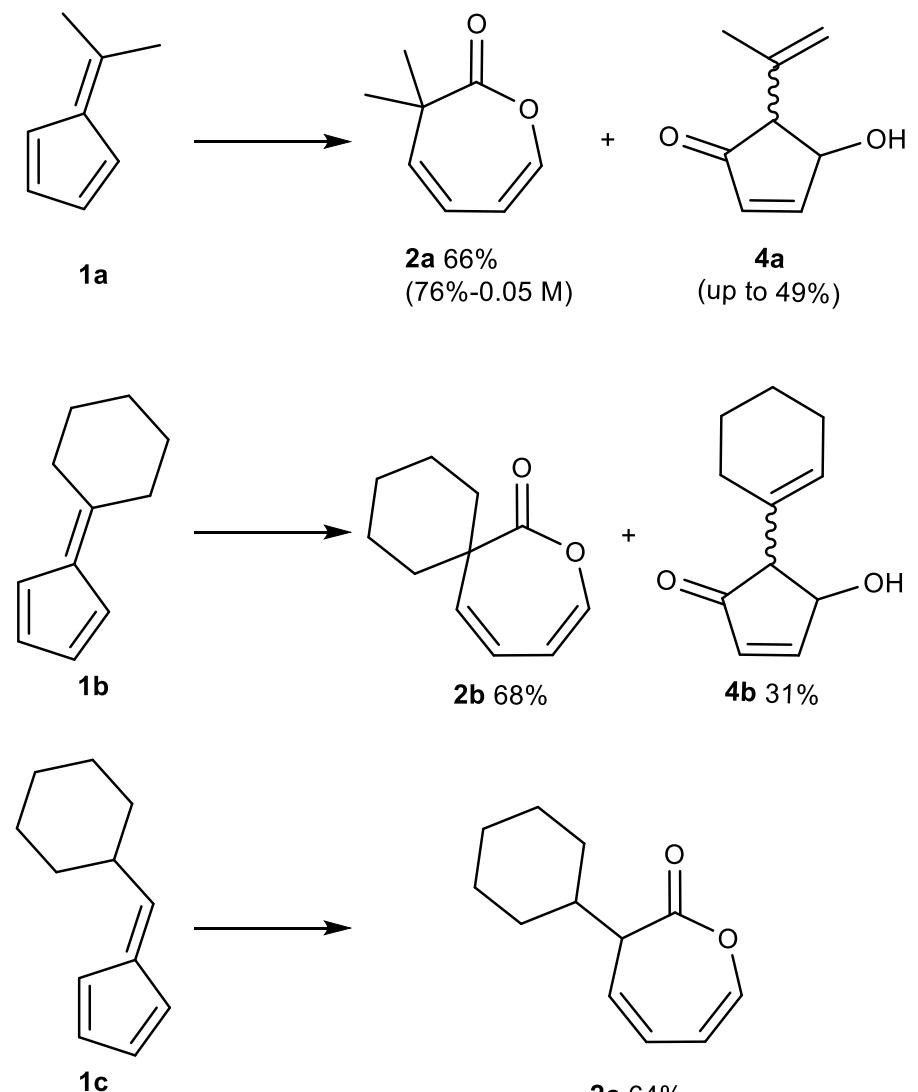

2c $64 \%$
$(66 \%-0.25 \mathrm{M})$
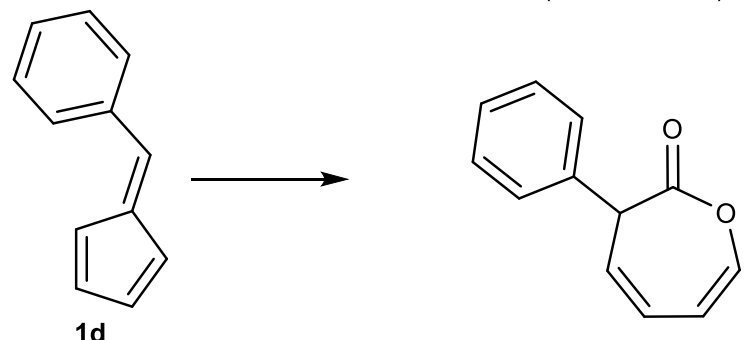

2d $70 \%$
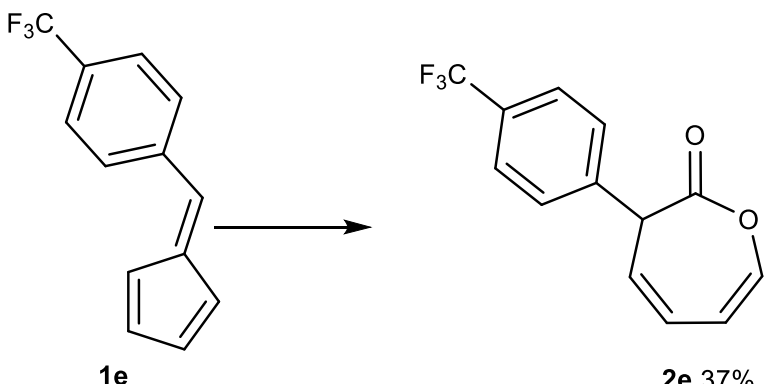

Scheme 3. Substrates run using the high-pressure photo-reactor with ${ }^{1} \mathrm{O}_{2}$ in $\mathrm{scCO}_{2}$ and the products formed. Yields were determined by ${ }^{1} \mathrm{H}$ NMR against a standard of mesitylene.

Furthermore, for a substrate containing an electron donating group (1f), the reaction was complicated by the formation of a polymeric mixture which fouled the photoreactor and led to 
blockages when EtOAc was used as a solvent (Scheme 4). These blockages could be prevented by using a 1:1 (v:v) mixture of EtOAc:MeOH; however useful yields of $2 \mathbf{f}$ could not be obtained $(<12 \%)$ and the material could not be isolated.

Recently, Erden and Gronert et al. demonstrated that fulvenes containing aromatic hydroxyl groups can be used to form 2-Coumaranone (5). ${ }^{[23]}$ In our reactor, this type of substrate (19) was reacted with complete conversion of the starting material. However, much like substrate 1f, the reaction again resulted in the formation of large quantities of polymeric material in the photoreactor with low yields of $\mathbf{5}$, along with a small amount of the lactone product $\mathbf{2} \mathbf{g}$ (Scheme 4). Again, the materials could not be isolated.

Supercritical $\mathrm{CO}_{2}$ is relatively non-polar in terms of its properties as a solvent; therefore, it is possible that, for some of the substrates containing more polar heteroatomic functional groups $(1 \mathrm{e}-\mathrm{g})$, the products or intermediates formed in the photoreactor are less soluble or even insoluble in $\mathrm{CO}_{2}$ and are precipitating within the photoreactor, hence leading to polymeric material and blockages. No definite identification of the solid material from the reactor fouling and blockages could be obtained when analysis was attempted.
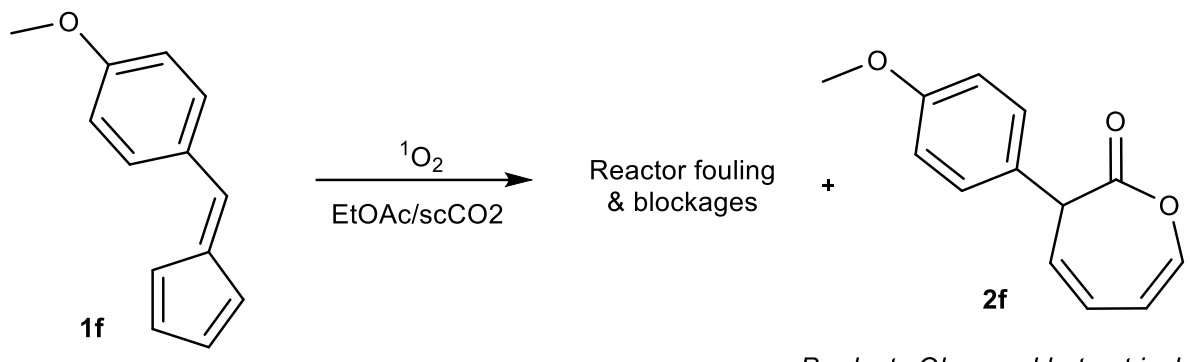

Products Observed but not isolated<smiles>Oc1ccccc1C=C1C=CC=C1</smiles>
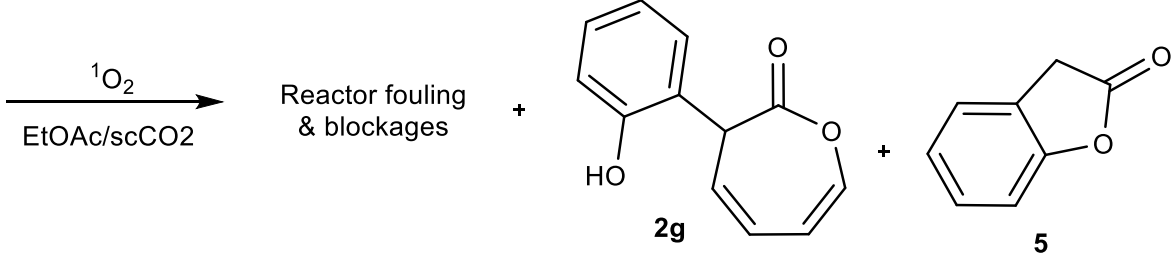

Scheme 4. Reactions of substrates containing electron donating groups (1f-g).

For all products of type 2 (2c-e) that were derived from fulvenes (1c-e), i.e. containing an $\mathrm{H}$ atom at the 3-position, prolonged exposure to silica or alumina led to isomerisation of the diene into conjugation with the carbonyl. However, flash chromatography on a short timescale could be used to purify these compounds before significant isomerisation occurred $(<10 \%)$.

To briefly test the reactivity of the lactone $\mathbf{2}$, small scale reactions were carried out. Hydrogentation of the lactones 2 yielded unsaturated 7-membered rings, which have potential 
value as functionalised polycaprolactone monomers (Scheme 5). Taking $\mathbf{2 c}$ and submitting it to a $\mathrm{Pd} / \mathrm{C}$ catalyst in EtOAc under an atmosphere of $\mathrm{H}_{2}$ yielded pure 6, which could be isolated without any further purification. Hydrogenation in $\mathrm{scCO}_{2}$ has been reported extensively by our group, ${ }^{[33-40]}$ however the coupling of a supercritical photo-oxidation reactor with a supercritical hydrogenation reactor presents several challenges not least the possibility of having high pressure mixtures of $\mathrm{H}_{2}$ and $\mathrm{O}_{2}$. Nevertheless, we aim to explore the streamlining of these process in the future.

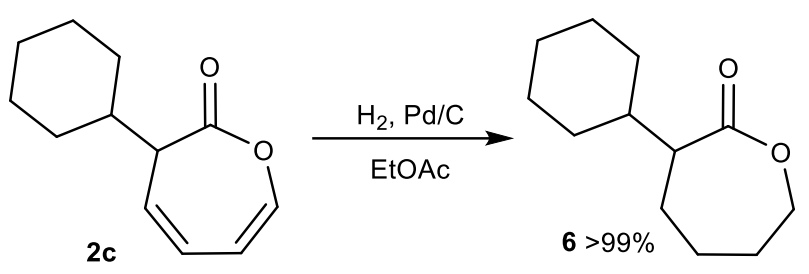

Scheme 5. Quantitative hydrogenation of $2 c$ to 6.

Submitting 2c to another round of ${ }^{1} \mathrm{O}_{2}$ did not yield any further reaction This supports the aparent stability of the lactones to further photo-oxidation observed in the first pass through the photoreactor, because no further oxidation products were observed in any of the continuous flow experiments. Furthermore, Diels-Alder reactions with 1,4-benzoquinone in toluene did not proceed with the diene when stirred at room temperature for $16 \mathrm{~h}$. Heating the mixture at reflux for $24 \mathrm{~h}$ also gave no conversion, and the starting materials were obtained unchanged.

\section{Conclusion}

We have applied a high pressure continuous flow photoreactor to the photo-oxidation of fulvenes. By using the continuous flow reactor with $\mathrm{CO}_{2}$ as an inert solvent, the photooxidation of several fulvenes was achieved. The coupling of a thermal reactor to the high pressure photoreactor allowed for efficient transformation of the reactive peroxide intermediates formed by reaction with ${ }^{1} \mathrm{O}_{2}$. The model substrate 6,6-dimethylfulvene (1a) showed interesting reactivity forming different compounds via competing pathways, the ratios of which depended on both the co-solvent choice and the flow rate of $\mathrm{CO}_{2}$. Other substrates that lacked the allylic hydrogen atoms of the model substrate gave the desired lactones exclusively as the major product. However more polar functional groups led to processing issues as reactor fouling and 
blockages occurred. One lactone was hydrogenated using $\mathrm{Pd} / \mathrm{C}$ demonstrating a potentially useful route to functionalised caprolactone derivates.

\section{Experimental}

CAUTION! The experiments described in this paper involve the use of relatively high pressures and require equipment with the appropriate pressure rating and with due regard to the potentially explosive reaction between $\mathrm{O}_{2}$ and organic compounds. Further details of the experiments carried out, the high-pressure equipment used, and the compound characterisation and spectra are provided in the Electronic Supporting Information.

\section{Acknowledgements}

We thank EPSRC grant (EP/P013341/1) supporting this work and the University of Nottingham Vice-Chancellor's Scholarship Award for funding the studentship for L.W. We also thank the analytical services at the University of Nottingham and M. Dellar, M.Guyler, D. Lichfield, R. Meehan and R. Wilson for their technical support.

\section{References}

[1] A. A. Ghogare, A. Greer, Chem. Rev. 2016, 116, 9994.

[2] P. R. Ogilby, Photochem. Photobiol. Sci. 2010, 9, 1543.

[3] R. Schmidt, Photochem. Photobiol. 2006, 82, 1161.

[4] M. C. DeRosa, R. J. Crutchley, Coord. Chem. Rev. 2002, 233, 351.

[5] A. G. Griesbeck, S. Sillner, M. Kleczka, in Singlet Oxygen: Applications in Biosciences and Nanosciences, Volume 1, Vol. 1, The Royal Society of Chemistry, 2016, pp. 369.

[6] J. Turconi, F. Griolet, R. Guevel, G. Oddon, R. Villa, A. Geatti, M. Hvala, K. Rossen, R. Goller, A. Burgard, Org. Process Res. Dev. 2014, 18, 417.

[7] O. Shvydkiv, C. Limburg, K. Nolan, M. OelgemÃ fller, J. Flow Chem. 2012, 2, 52.

[8] G. I. Ioannou, T. Montagnon, D. Kalaitzakis, S. A. Pergantis, G. Vassilikogiannakis, ChemPhotoChem 2017, 1, 173.

[9] A. Kouridaki, K. Huvaere, React. Chem. Eng. 2017, 2, 590.

[10] D. S. Lee, Z. Amara, C. A. Clark, Z. Y. Xu, B. Kakimpa, H. P. Morvan, S. J. Pickering, M. Poliakoff, M. W. George, Org. Process Res. Dev. 2017, 21, 1042.

[11] C. A. Clark, D. S. Lee, S. J. Pickering, M. Poliakoff, M. W. George, Org. Process Res. Dev. 2016, 20, 1792. 
[12] F. Levesque, P. H. Seeberger, Org. Lett. 2011, 13, 5008.

[13] X. Han, R. A. Bourne, M. Poliakoff, M. W. George, Green Chem. 2009, 11, 1787.

[14] R. A. Bourne, X. Han, M. Poliakoff, M. W. George, Angew. Chem. Int. Ed. 2009, 48, 5322.

[15] Z. Amara, J. F. B. Bellamy, R. Horvath, S. J. Miller, A. Beeby. A. Burgard, K. Rossen, M. Poliakoff, M. W. George, Nat. Chem. 2015 7, 489.

[16] J. F. Hall, X. Han, M. Poliakoff, R. A. Bourne, M. W. George, Chem. Commun. 2012, 48, 3073.

[17] X. Han, R. A. Bourne, M. Poliakoff, M. W. George, Chem. Sci. 2011, 2, 1059.

[18] R. A. Bourne, X. Han, A. O. Chapman, N. J. Arrowsmith, H. Kawanami, M. Poliakoff, M. W. George, Chem. Commun. 2008, 4457.

[19] D. R. Worrall, A. A. Abdel-Shafi, F. Wilkinson, The Journal of Physical Chemistry A 2001, 105, 1270.

[20] L. Wu, Z. Abada, D. S. Lee, M. Poliakoff, M. W. George, Tetrahedron 2018, DOI: 10.1016/j.tet.2017.11.048.

[21] M. B. Plutschack, B. Pieber, K. Gilmore, P. H. Seeberger, Chem. Rev. 2017, 117, 11796.

[22] W. R. Adams, D. J. Trecker, Tetrahedron 1971, 27, 2631.

[23] I. Erden, J. Basada, D. Poli, G. Cabrera, F. P. Xu, S. Gronert, Tetrahedron Lett. 2016, $57,2190$.

[24] N. Harada, H. Uda, H. Ueno, Chem Lett 1972, 663.

[25] N. Harada, S. Suzuki, H. Ueno, H. Uda, J. Am. Chem. Soc. 1972, 94, 1777.

[26] N. Harada, S. Suzuki, H. Uda, H. Ueno, Chem. Lett. 1972, 803.

[27] W. Skorianetz, Schultee.Kh, G. Ohloff, Helv. Chim. Acta 1971, 54, 1913.

[28] N. Coskun, I. Erden, Tetrahedron 2011, 67, 8607.

[29] C. Reynaud, Y. Fall, M. Feuerstein, H. Doucet, M. Santelli, Tetrahedron 2009, 65, 7440.

[30] S. T. Staben, L. H. Xin, F. D. Toste, J. Am. Chem. Soc. 2006, 128, 12658.

[31] I. Erden, N. Ocal, J. G. Song, C. Gleason, C. Gartner, Tetrahedron 2006, 62, 10676.

[32] It is possible that $\mathrm{NEt}_{3}$, could have inhibited the formation of ${ }^{1} \mathrm{O}_{2}$, thereby reducing the efficiency of the reaction.

[33] Z. Amara, M. Poliakoff, R. Duque, D. Geier, G. Francio, C. M. Gordon, R. E. Meadows, R. Woodward, W. Leitner, Org. Process Res. Dev. 2016, 20, 1321.

[34] X. Han, M. Poliakoff, Chem. Soc. Rev. 2012, 41, 1428.

[35] J. G. Stevens, P. Gomez, R. A. Bourne, T. C. Drage, M. W. George, M. Poliakoff, Green Chem. 2011, 13, 2727. 
[36] J. G. Stevens, R. A. Bourne, M. V. Twigg, M. Poliakoff, Angew. Chem. Int. Ed. 2010, 49, 8856.

[37] R. A. Bourne, J. G. Stevens, J. Ke, M. Poliakoff, Chem. Commun. 2007, 4632.

[38] P. Stephenson, P. Licence, S. K. Ross, M. Poliakoff, Green Chem., 2004, 6, 521.

[39] P. Licence, J. Ke, M. Sokolova, S. K. Ross, M. Poliakoff, Green Chem., 2003, 5, 99.

[40] M. G. Hitzler, F. R. Smail, S. K. Ross, M. Poliakoff, Org. Process Res. Dev. 1998, 2, 137.

\section{TOC Graphic}
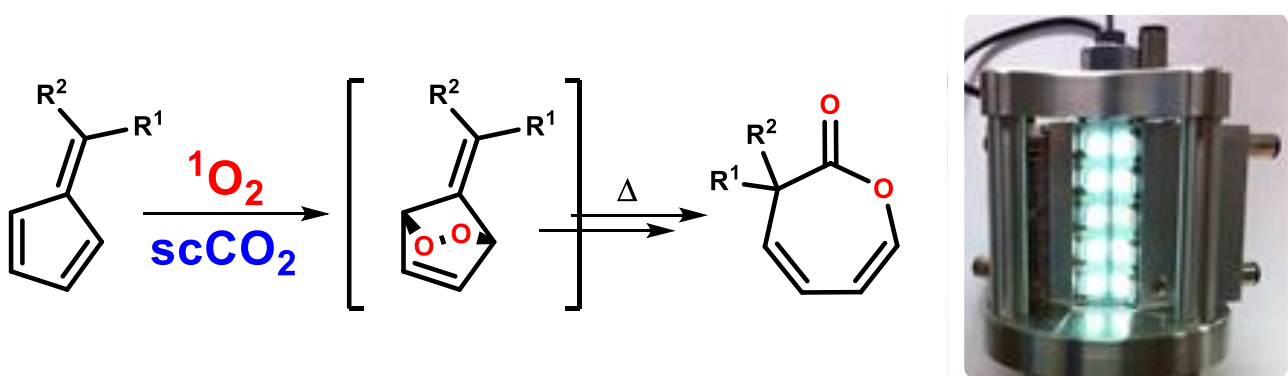

The continuous photo-oxidation of fulvenes is realised using a high pressure photoreactor using supercritical carbon dioxide $\left(\mathrm{scCO}_{2}\right)$ as a solvent. The lactone products can be formed in high yield depending on functionality. Further reactivity of the lactones have been explored. 